\title{
4-PLANAR GEODESIC KAEHLER IMMERSIONS INTO A COMPLEX PROJECTIVE SPACE
}

\author{
JIN SUK PAK AND KUNIO SAKAMOTO \\ (Communicated by David G. Ebin)
}

\begin{abstract}
If $f$ is a proper 4-planar geodesic Kaehler immersion of a connected complete Kaehler manifold $M^{n}(n \geq 2)$ into $C P^{m}(c)$, then $M^{n}=$ $C P^{n}(c / 4)$ and $f$ is equivalent to the 4 th Veronese map.
\end{abstract}

0. Introduction. Let $\bar{M}$ be a Riemannian manifold. A curve $\tau: I \rightarrow \bar{M}$ defined on an open interval $I$ is said to be $d$-planar if there exist an open interval $I_{s}(s \in$ $\left.I_{s} \subset I\right)$ and a $d$-dimensional totally geodesic submanifold $P_{s}$ for each $s \in I$ such that $\tau\left(I_{s}\right) \subset P_{s}$. Moreover, a $d$-planar curve $\tau$ is said to be proper if it is not $(d-1)$-planar on each open subinterval of $I$. An isometric immersion $f: M \rightarrow \bar{M}$ of a Riemannian manifold $M$ is called a (resp. proper) $d$-planar geodesic immersion if $\tau=f \circ \gamma$ is (resp. proper) $d$-planar geodesic for every geodesic $\gamma: I \rightarrow M$. 1-planar geodesic immersions are totally geodesic. 2-planar geodesic immersions into real space forms were classified in [7] (for other treatment, see [1]).

When the ambient manifold is a complex projective space $C P^{m}(c)$ with constant holomorphic sectional curvature $c$, 2-planar and odd order proper planar geodesic Kaehler immersions were classified in [5 and 6], respectively. In this paper, we shall study proper 4-planar geodesic Kaehler immersions into $C P^{m}(c)$.

1. Notation and basic equations (cf. [2]). For a Kaehler immersion $f: M \rightarrow$ $C P^{m}(c)$, the second fundamental form and Weingarten map corresponding to a normal vector field $\xi$ will be denoted by $H$ and $A_{\xi}$, respectively. Gauss and Weingarten's equations are given by

$$
\bar{\nabla}_{X} Y=\nabla_{X} Y+H(X, Y), \quad \bar{\nabla}_{X} \xi=-A_{\xi} X+\nabla_{X}^{\perp} \xi
$$

for all tangent vector fields $X$ and $Y$ on $M$, where $\bar{\nabla}, \nabla$, and $\nabla^{\perp}$ denote the covariant differentiation of $\bar{M}, M$, and the normal bundle, respectively. Let $R$ be the curvature tensor and $J$ the complex structure of $M$. The structure equation of Gauss is given by

$$
\begin{aligned}
R(X, Y) Z & \\
= & (c / 4)\{\langle Y, Z\rangle X-\langle X, Z\rangle Y+\langle J Y, Z\rangle J X-\langle J X, Z\rangle J Y-2\langle J X, Y\rangle J Z\} \\
& +A_{H(Y, Z)} X-A_{H(X, Z)} Y .
\end{aligned}
$$

Received by the editors January 20, 1987.

1980 Mathematics Subject Classification (1985 Revision). Primary 53C40. forms.

Key words and phrases. 4-planar geodesic immersions, Kaehler manifolds, second fundamental

Research partially supported by grant from Korea Science and Engineering Foundation. 
The structure equation of Codazzi reduces to $(D H)(X, Y, Z)=(D H)(Y, X, Z)$, where

$$
(D H)(X, Y, Z)=\nabla_{X}^{\perp} H(Y, Z)-H\left(\nabla_{X} Y, Z\right)-H\left(Y, \nabla_{X} Z\right) .
$$

Let $\bar{J}$ be the complex structure of $C P^{m}(c)$. Since $\overline{\nabla J}=0$, we have

$$
H(J X, Y)=\bar{J} H(X, Y) .
$$

If $H$ satisfies $\|H(X, X)\|^{2}=\lambda^{2}(x)$ for all unit vectors $X \in T_{x} M$ and each $x \in M$, then the immersion $f$ is said to be isotropic (or $\lambda$-isotropic). We note that $f$ is isotropic if and only if $\langle H(X, X), H(X, Y)\rangle=0$ for any orthonormal vectors $X$ and $Y$ at every point.

2. Proper 4-planar geodesic Kaehler immersions. Let $M$ be a connected complete Riemannian manifold and $f: M \rightarrow C P^{m}(c)$ a proper $d$-planar geodesic immersion. We first prove

LEMMA 2.1. For each geodesic $\gamma$ of $M$, there exists a unique d-dimensional totally geodesic submanifold $P_{\gamma}$ such that $\tau((-\infty, \infty)) \subset P_{\gamma}$, where $\tau=f \circ \gamma$. Each $P_{\gamma}$ is complex or totally real.

ProOF. Let $u \in(-\infty, \infty)$ be arbitrarily fixed and put $P_{\gamma}=P_{u}$. If we define a set $U$ by $U=\left\{s \in(-\infty, \infty): \tau(s) \in P_{\gamma}\right\}$, then $U$ is nonempty and closed. Let $v \in U$. Consider a finite cover $\left\{I_{s_{1}}=I_{u}, I_{s_{2}}, \ldots, I_{s_{k}}=I_{v}\right\}$ of $[u, v]$ where we have assumed $u<v$ without loss of generality. Noting that $f$ is proper $d$-planar geodesic and the intersection of two totally geodesic submanifolds is a totally geodesic submanifold, we see that $P_{\gamma}=P_{u}=P_{s_{1}}=\cdots=P_{v}$. Therefore, $I_{v} \subset U$, i.e., $U$ is open and hence $U=(-\infty, \infty)$. The uniqueness of $P_{\gamma}$ is easily derived from the assumption that $f$ is proper $d$-planar geodesic. It is well known that a submanifold in $C P^{m}(c)$ is complex or totally real if and only if the second fundamental form $H$ of the submanifold satisfies $(D H)(X, Y, Z)=(D H)(Y, X, Z)$ (cf. [6, (1.9), p. 300]). Therefore, since $P_{\gamma}$ is totally geodesic, we have the assertion. Q.E.D.

Let $x \in M, X \in U_{x} M$ (unit tangent sphere at $x$ ), and let $\gamma$ be the unit speed geodesic such that $\gamma(0)=x$ and $\dot{\gamma}(0)=X$. Then $\tau=f \circ \gamma$ satisfies

$$
\begin{aligned}
\dot{\tau}(0) & =f_{*} X, \\
\bar{\nabla}_{X} \dot{\tau} & =H(X, X), \\
\bar{\nabla}_{X}^{2} \dot{\tau} & =-A_{H(X, X)} X+(D H)(X, X, X) .
\end{aligned}
$$

Higher order covariant derivatives of $\dot{\tau}$ in the direction $X$ can be also obtained by using Gauss and Weingarten equations (1.1). Note that all covariant derivatives of $\dot{\tau}$ are tangent to $P_{\gamma}$. Define a function $\phi$ on the unit tangent sphere bundle $U M$ over $M$ by

$$
\begin{aligned}
\phi(X) & =\operatorname{det}\left(\left\langle\bar{\nabla}_{X}^{i} \dot{\tau}, \bar{\nabla}_{X}^{j} \dot{\tau}\right\rangle_{i, j=0,1, \ldots, d-1}\right) \\
& =\text { Gramian of vectors } X, \bar{\nabla}_{X} \dot{\tau}, \ldots, \bar{\nabla}_{X}^{d-1} \dot{\tau}
\end{aligned}
$$

for $X \in U M$. If $\phi(X) \neq 0$, then vectors $X, \bar{\nabla}_{X} \dot{\tau}, \ldots, \bar{\nabla}_{X}^{d-1} \dot{\tau}$ form a base of $T_{x} P_{\gamma}$. 
LEMMA 2.2. Let $S$ be any connected component of the set $\{X \in U M: \phi(X) \neq$ $0\}$. Then $P_{\gamma^{x}}$ is complex for every $X \in S$ or totally real for every $X \in S$ where $\gamma^{X}$ denotes the geodesic tangent to $X$.

Proof. Assume that there exist $X$ and $Y$ in $S$ such that $P_{\gamma^{x}}$ is complex and $P_{\gamma^{Y}}$ is totally real. Since $S$ is arcwise connected, there is a smooth curve $X(t)$ in $S$ such that $X(0)=X$ and $X(1)=Y$. Consider a function $\psi$ on $[0,1]$ defined by

$$
\psi(t)=\operatorname{Sup}\left\{\langle\bar{J} X(t), Z\rangle: Z \in T_{\pi(X(t))} P_{\gamma^{x(t)}},\|Z\|=1\right\},
$$

where $\pi: U M \rightarrow M$ is the projection. Since

$$
T_{\pi(X(t))} P_{\gamma^{X(t)}}=\operatorname{Span}\left\{X(t), \bar{\nabla}_{X(t)} \dot{\tau}_{t}, \ldots, \bar{\nabla}_{X(t)}^{d-1} \dot{\tau}_{t}\right\} \quad\left(\tau_{t}=f \circ \gamma^{X(t)}\right)
$$

which is a smooth curve in the Grassmann bundle of $d$-planes over $M$, we see that $\psi$ is a continuous function. Moreover, $P_{\gamma^{X(t)}}$ is complex or totally real (Lemma 2.1) and hence $\psi(t)=1$ or 0 for each $t \in[0,1]$. Thus $\psi$ is constant. However $\psi(0)=1$ and $\psi(1)=0$. Q.E.D.

Now we explain Kaehler immersions into $C P^{m}(c)$ of symmetric Kaehler manifolds of compact type. Let $M$ be an irreducible symmetric Kaehler manifold of compact type and $k$ a positive integer. In [4], Nakagawa and Takagi constructed a full equivariant Kaehler imbedding $f_{k}: M \rightarrow C P^{m}(c)$ which is called the $k$ th full Kaehler imbedding of $M$. Moreover, in [8] Takagi and Takeuchi constructed a full Kaehler imbedding of a (not necessarily irreducible) symmetric Kaehler manifold $M$ of compact type into $C P^{m}(c)$ as follows. Let $M_{i}(i=1, \ldots, q)$ be the irreducible components of $M$, i.e., $M=M_{1} \times \cdots \times M_{q}$ and $f_{k_{i}}: M_{i} \rightarrow$ $C P^{m_{i}}(c)$ be the $k_{i}$ th full Kaehler imbedding of $M_{i}$. Define a full Kaehler imbed$\operatorname{ding} S_{q}: C P^{m_{1}}(c) \times \cdots \times C P^{m_{q}}(c) \rightarrow C P^{m}(c)$ by the multifold tensor product of the homogeneous coordinates where $m=\left(m_{1}+1\right) \times \cdots \times\left(m_{q}+1\right)-1$ and we notice that $S_{2}$ is the Segre imbedding. Then $S_{q} \circ\left(f_{k_{1}} \times \cdots \times f_{x_{q}}\right)$ becomes a full equivariant Kaehler imbedding of $M$ into $C P^{m}(c)$. In [4 and 9], it was shown that any full Kaehler immersion of a compact symmetric Kaehler manifold into $C P^{m}(c)$ is obtained in this way. In particular, if $M=C P^{n}(c / k)$, then the $k$ th full Kaehler imbedding $V_{k}^{n}: C P^{n}(c / k) \rightarrow C P^{m(k)}(c)$ is called the kth Veronese map which is defined by

$$
\left[z_{i}\right]_{0 \leq i \leq n} \mapsto\left[\left(\frac{k !}{k_{0} ! \cdots k_{n} !}\right)^{1 / 2} z_{0}^{k_{0}} \cdots z_{n}^{k_{n}}\right]_{k_{0}+\cdots+k_{n}=k}
$$

where $[*]$ means the point of the projective space with the homogeneous coordinate * and $m(k)=\left(\begin{array}{c}n+k \\ k\end{array}\right)-1$.

The following two lemmas were proved in [6].

LEMMA 2.3 (CF. THE PROOF OF PROPOSITION 2.1 IN [6]). Let $f: M \rightarrow$ $C P^{m}(c)$ be a Kaehler immersion of a connected complete Kaehler manifold $M$ into $C P^{m}(c)$. Assume that $\langle H(X, X),(D H)(X, X, X)\rangle=0$ for every $X \in T M$. Then $M$ is a compact simply connected symmetric Kaehler manifold.

LEMMA 2.4 (CF. THE PROOF OF THEOREM 2.3 IN [6]). Let $f: M \rightarrow$ $C P^{m}(c)$ be a proper d-planar geodesic Kaehler immersion of a symmetric Kaehler 
manifold of compact type. Then $M^{n}=C P^{n}(c / d)$ and $f$ is equivalent to $i \circ V_{d}^{n}$, where $i: C P^{m(d)}(c) \rightarrow C P^{m}(c)$ is a totally geodesic imbedding.

Here we note that the equivalence of two isometric immersions $f$ and $f^{\prime}$ of a Riemannian manifold into a Riemannian manifold $\bar{M}$ is defined as follows: If there exists an isometry $F$ of $\bar{M}$ such that $f^{\prime}=F \circ f$, then $f$ and $f^{\prime}$ are said to be equivalent.

LEMMA 2.5. Let $f: M^{n} \rightarrow C P^{m}(c)$ be a Kaehler immersion of a connected complete Kaehler manifold $M^{n}$. Assume that $n \geq 2$ and $f$ is isotropic on a connected open subset $M_{0}$ in $M^{n}$. Then $M^{n}=C P^{n}(c / k)$ and $f$ is equivalent to $i \circ V_{k}^{n}$ for some $k$.

PROOF. Using (1.2), we see that the holomorphic sectional curvature of $M_{0}$ is equal to $c-2 \lambda^{2}$ where $\lambda^{2}=\|H(X, X)\|^{2}$. It follows from the holomorphic analogue of Schur's Theorem [2, Theorem 7.5, p. 168] that $M_{0}(n \geq 2)$ is a Kaehler manifold of constant holomorphic sectional curvature. Thus $M^{n}$ is also of constant holomorphic sectional curvature since $M^{n}$ is analytic. Hence we can conclude from [3] that $M^{n}=C P^{n}(c / k)$ and $f$ is equivalent to $i \circ V_{k}^{n}$ for some positive integer k. Q.E.D.

THEOREM 2.6. Let $f: M^{n} \rightarrow C P^{m}(c)$ be a proper 4-planar geodesic Kaehler immersion and $n \geq 2$. Then $M^{n}=C P^{n}(c / 4)$ and $f$ is equivalent to $i \circ V_{4}^{n}$, where $i: C P^{m(4)}(c) \rightarrow C P^{m}(c)$ is a totally geodesic imbedding.

REMARK. If $m(4)>m$, then such immersion does not exist.

PROOF. Assume that the set $S$ in Lemma 2.2 is not empty. By Lemma 2.2, there are two cases: (I) $P_{\gamma^{x}}$ is totally real for every $X \in S$, and (II) $P_{\gamma^{x}}$ is complex for every $X \in S$.

Case (I). Equation (2.1) implies that

$$
\langle\bar{J} H(X, X),(D H)(X, X, X)\rangle=0
$$

for every $X \in S$. Since the left-hand side of (2.2) is real analytic on $U M$ and $S$ is open, we see that (2.2) holds for every $X \in U M$. Using (1.3) and the Codazzi equation, we have

$$
(D H)(J Z, Y, X)=\bar{J}(D H)(Z, Y, X)
$$

for every $X, Y, Z \in T M$. Replacing $X$ by $J X$ in (2.2) and using (1.3) and (2.3), we have $\langle H(X, X),(D H)(X, X, X)\rangle=0$ for every $X \in T M$. Therefore, we conclude from Lemmas 2.3 and 2.4 that $M=C P^{n}(c / 4)$ and $f$ is equivalent to $i \circ V_{4}^{n}$.

Case (II). Since $\phi(X) \neq 0$ on $S, H(X, X) \neq 0$ for every $X \in S$. Thus vectors $X, J X, H(X, X)$, and $\bar{J} H(X, X)$ span $T_{\pi(X)} P_{\gamma^{X}}$. It follows from (2.1) that

$$
\langle H(X, X), H(X, Y)\rangle=\left\langle A_{H(X, X)} X, Y\right\rangle=0
$$

for any $Y \in T_{\pi(X)} M$ orthogonal to $\operatorname{Span}\{X, J X\}(X \in S)$. Furthermore, we have

$$
\langle H(X, X), H(X, J X)\rangle=\langle H(X, X), \bar{J} H(X, X)\rangle=0 .
$$

Therefore, (2.4) holds for every $Y \in T_{\pi(X)} M$ orthogonal to $X \in S$. In other words, the function $\lambda^{2}: X \mapsto\|H(X, X)\|^{2}$ defined on $U M$ has the vanishing derivative in the direction of the fibre on $U_{\pi(X)} M \cap S(X \in S)$. Since $U_{\pi(X)} M \cap S$ is open and $\lambda^{2}$ 
is real analytic, $\lambda^{2}$ is constant on $U_{\pi(X)} M$ for $X \in S$. Thus $f$ is $\lambda$-isotropic on the connected open subset $M_{0}=\pi(S)$. We see from Lemma 2.5 that $M^{n}=C P^{n}(c / k)$ and $f$ is equivalent to $i \circ V_{k}^{n}$ for some positive integer $k$. The $k$ th Veronese map $V_{k}^{n}: C P^{n}(c / k) \rightarrow C P^{m(k)}(c)$ is proper $k$-planar geodesic. However, $P_{\gamma}$ is totally real for every geodesic $\gamma$ in $C P^{n}(c / k)$ (cf. [6, Lemma 2.2 and its proof, p. 303]). Hence this case does not occur.

Next let us assume that $\phi=0$ on $U M$. Suppose that there exists a geodesic $\gamma$ such that $P_{\gamma}$ is totally real. Then the order of $f \circ \gamma$ is not greater than 3 , and hence an open segment $(f \circ \gamma)(I)$ is contained in a 3-dimensional totally geodesic submanifold of $P_{\gamma}=R P^{3}(c / 4)$ (for the definition of the order of a curve, see [6]). This contradicts the assumption that $f$ is proper 4-planar geodesic. Thus $P_{\gamma}$ is a complex totally geodesic submanifold for every $\gamma$. If $f$ is not totally geodesic, then the function $\lambda^{2}$ does not vanish identically on $U M$. Thus if we define $S$ by a connected component of the set $\{X \in U M, \lambda(X) \neq 0\}$ and $M_{0}=\pi(S)$, then, using the same argument as Case (II), we have a contradiction. Q.E.D.

REMARK. We have used the condition that $f$ is 4-planar geodesic in order to prove $\left\langle\bar{\nabla}_{X}^{2} \dot{\tau}, Y\right\rangle=0$ for every $Y$ orthogonal to $\operatorname{Sp}\{X, J X\}$. There is a conjecture that if $f: M \rightarrow C P^{m}(c)$ is a proper $d$-planar geodesic Kaehler immersion, where $d$ is even, then $M=C P^{n}(c / d)$ and $f$ is equivalent to $V_{d}^{n}$.

It seems to be interesting that we characterize Kaehler immersions of compact symmetric Kaehler manifolds by the shape of geodesics.

The following is an easy consequence of Lemma 2.3.

PROPOSITION 2.7. Let $f: M \rightarrow C P^{m}(c)$ be a Kaehler immersion of a connected complete Kaehler manifold $M$. The first Frenet curvature of $\tau=f \circ \gamma$ is constant along $\tau$ for every geodesic $\gamma$ of $M$ if and only if $M$ is a compact simply connected symmetric Kaehler manifold and $f$ is equivalent to a full equivariant Kaehler imbedding mentioned before in this section.

\section{REFERENCES}

1. B. Y. Chen and P. Verheyen, Submanifolds with geodesic normal sections, Math. Ann. 269 (1984), 417-429.

2. S. Kobayashi and K. Nomizu, Foundations of differential geometry. II, Interscience, New York, 1969.

3. H. Nakagawa and K. Ogiue, Complex space forms immersed in complex space forms, Trans. Amer. Math. Soc. 219 (1976), 289-297.

4. H. Nakagawa and R. Takagi, On locally symmetric Kaehler submanifolds in a complex projective space, J. Math. Soc. Japan 28 (1976), 638-667.

5. J. S. Pak, Planar geodesic submanifolds in complex space forms, Kodai Math. J. 1 (1978), 187-196.

6. J. S. Pak and K. Sakamoto, Submanifolds with proper d-planar geodesics immersed in complex projective spaces, Tôhoku Math. J. 38 (1986), 297-311.

7. K. Sakamoto, Planar geodesic immersions, Tôhoku Math. J. 29 (1977), 25-56.

8. R. Takagi and M. Takeuchi, Degree of symmetric Kaehlerian submanifolds of a complex projective space, Osaka J. Math. 14 (1977), 501-518.

9. M. Takeuchi, Homogeneous Kaehler submanifolds in complex projective spaces, Japan. J. Math. 4 (1978), 171-219.

Department of Mathematics, Teachers College, KyUngPoOK National UniVERSITY, TAEGU 702-701, KOREA

DEPARTMENT OF MATHEMATICS, TOKYo InSTitute of TECHNOLOGy, OHOKAyAMA, MEGURO-KU, TOKYO 152, JAPAN 